\title{
Concurrent palliative external radiotherapy with sorafenib or doxorubicin for bulky differentiated thyroid carcinoma: A case report
}

\author{
KAREN S. CORTÉS-MATEUS ${ }^{1}$, KATARZYNA HOLUB ${ }^{1}$, FABRICIO RACCA ${ }^{2}$, \\ JUAN JOSÉ GRAU ${ }^{1}$ and JAUME CAPDEVILA ${ }^{2}$ \\ ${ }^{1}$ Medical Oncology Department and Radiation Oncology Department, University of Barcelona, \\ IDIBAPS, Hospital Clinic Barcelona, 08036 Barcelona; ${ }^{2}$ Vall Hebron University Hospital, \\ Vall Hebron Institute of Oncology (VHIO), 08035 Barcelona, Spain
}

Received September 7, 2017; Accepted June 19, 2018

DOI: $10.3892 / \mathrm{ol} .2018 .9069$

\begin{abstract}
Administration of external beam radiotherapy (EBRT) for a bulky recurrence or primary bulky tumor of differentiated thyroid carcinoma (DTC) is rare. No previous experience is available on the feasibility of administering EBRT simultaneously with systemic treatment with doxorubicin or sorafenib, or both. The present case study reported the results from two different institutions on 5 consecutive patients. Side effects and tolerance to radiotherapy plus systemic treatment with doxorubicin $\left(20 \mathrm{mg} / \mathrm{m}^{2}\right.$ intravenously weekly for $6 / 8$ consecutive weeks) or sorafenib $(400 \mathrm{mg} / 12 \mathrm{~h}$ orally for 8 weeks) or both, were analyzed in patients with DTC. The local response to radiotherapy and patient outcome was also analyzed. A total of 4 males and 1 female, aged 37-62 years with cervical bulky mass DTC were included. The pathological tumor types were papillary (2 patients), follicular (2 patients) and medullar thyroid carcinoma (1 patient). The radiated cervical mass was local recurrence in 3 cases and primary tumor in the other two. The total dose of radiotherapy ranged between 50 and $64.8 \mathrm{~Gy}$. Three patients received sorafenib, 1 patient received doxorubicin and 1 patient received both treatments. The total planned dose of radiotherapy was administered to all patients. Grade 2 anemia and erythrodysesthesia was the most frequent toxicity. Only the patient who received doxorubicin plus sorafenib had grade 3 toxicity consisting of lymphopenia, folliculitis and mucositis. All but 1 patient had a good local response to radiotherapy. The administration of EBRT concurrently with sorafenib and doxorubicin to patients with DTC with a bulky cervical mass is feasible.
\end{abstract}

Correspondence to: Dr Juan José Grau, Medical Oncology Department and Radiation Oncology Department, University of Barcelona, IDIBAPS, Hospital Clinic Barcelona, 170 Villarroel Street, 08036 Barcelona, Spain

E-mail: jjgrau@clinic.cat

Key words: thyroid cancer, sorafenib, doxorubicin, toxicity, tolerance

\section{Introduction}

Differentiated thyroid carcinoma (DTC) is the most common endocrine malignancy (1). Standard treatment is surgery and hormone suppression with levothyroxine, followed by treatment with radioiodine $\mathrm{I}^{-131}$ (Thyroid carcinoma. NCCN Clinical Practice Guidelines in Oncology) (2).

Sometimes DTC is classified as 'bulky' at presentation, because of a bulky primary tumor or voluminous local recurrence or lymph node involvement. In that case, the patient may have dyspnea and stridor due to compression of the larynx or trachea, requiring surgical intervention. Tumor size and infiltration of neighboring organs such as the larynx can mean that surgery is not always feasible in such situations.

According to the latest American Thyroid Association (ATA) 2015 guidelines, bulky tumor recurrence is one of the causes of radioiodine resistance, both in bulky tumor recurrences and primary tumor presence, mainly because the unresectability of the primary thyroid gland prevents the administration of radioactive iodine therapy (2). When radioiodine resistance occurs, treatment with external beam radiotherapy (EBRT) for locoregional control and symptomatic palliation is indicated, as $50 \%$ of patients have compromised airways $(3,4)$. External beam radiation may be ineffective and highly toxic if the tumor is very large. Therefore, palliative therapy with doxorubicin may be indicated to improve outcome in some institutions (5-8). In other tumors, such as non-small cell lung carcinoma, palliative systemic treatment with multikinase inhibitors has been indicated in cases of metastatic disease, concomitantly with external radiation therapy. The combination of multikinase inhibitors and external radiation therapy was seen to be feasible and tolerance was acceptable (9). However, the experience of concomitant therapy (radiotherapy plus targeted agent) in locally advanced cancers is scant, although synergistic effects have already been described in some other tumor types $(9,10)$.

The use of sorafenib in radioiodine-resistant DTC has been approved since 2014 (11), although it was previously administered in compassionate use programs for tumors with rapid progression or poor response to treatment (12). It remains unclear whether the association with external radiotherapy is 
possible, and if tolerance, toxicity and efficacy are clinically acceptable.

We present the tolerance, toxicity and clinical activity results of 5 consecutive patients who were seen in the emergency room (ER) of 2 different institutions for dyspnea and stridor due to thyroid cancer. All fulfilled criteria that precluded surgical treatment, and consequently were prescribed concomitant treatment with local external radiation therapy and systemic treatment with doxorubicin or sorafenib. Patients were treated according to best practice protocols, and all procedures met the ethical principles of the declaration of Helsinki. The main toxicities for each patient are shown in Table I.

\section{Case report}

Patient 1. A 50-year-old man presented in the ER for dyspnea and stridor caused by a bulky left cervical mass. He was in good health, smoker of 9 packs/year, and was receiving simvastatin for dyslipidemia. The patient had been diagnosed with papillary thyroid carcinoma (PTC) T4NOM0 two years previously. Treatment consisted of total thyroidectomy, revealing an $\mathrm{R} 1$ (microscopic margin affected by the tumor) at the tracheal surgical margin, followed by $100 \mathrm{mCi}$ radioiodine. The patient was asymptomatic after treatment. The disease progressed 1 year before the ER consultation in the form of lung metastases and cervical lymph node recurrence which was treated with adenectomy followed by radioiodine $100 \mathrm{mCi}$. Seven months later he presented cervical relapse and progression of the pulmonary metastases.

The cervical mass grew rapidly within a few weeks precipitating the ER consultation; positron emission tomography/computed tomography (PET-CT) confirmed cervical, mediastinal and pulmonary progression (Fig. 1, left). Palliative external RT beam (50 Gy) was administered with a boost of 20 Gy along with concomitant doxorubicin $\left(20 \mathrm{mg} / \mathrm{m}^{2}\right.$ per week) for 4 weeks plus sorafenib $(400 \mathrm{mg} / 12 \mathrm{~h})$, achieving partial response (Fig. 1, right) (13). Adverse effects to treatment included grade 2 anemia, grade 1 renal failure, and hypocalcemia, grade 2 mucositis, and esophagitis that improved within a few weeks of symptomatic treatment. After 6 months, he presented progression of pulmonary metastases and was treated in an investigational trial of lenvatinib. He died 59 months after radiotherapy due to pulmonary progression.

Patient 2. A 58-year-old man, former smoker with no other medical history of interest, presented in the ER due to dyspnea and stridor caused by a fast-growing right cervical mass. The CT scan showed a $44 \times 47 \mathrm{~mm}$ mass at the right thyroid lobe and fine needle aspiration (FNA) was positive for follicular thyroid carcinoma (FTC); staging after subtotal thyroidectomy was pT4N3M0. In the follow-up, PET-CT showed persistent disease with significant heterogeneous uptake (maximum SUV 37.8), suggesting that the patient would not benefit from treatment with I-131.

Two months after surgery, he presented rapid growth of the residual mass, which reached dimensions of $60 \times 94 \times 68 \mathrm{~mm}$ with multiple lymph nodes in levels II, III and IV (Fig. 2, left). Because of the rapid progression and radioiodine resistance, he received external RT at a total dose (TD) of $50 \mathrm{~Gy}$ concomitantly with doxorubicin $\left(20 \mathrm{mg} / \mathrm{m}^{2}\right.$ per week for 6 weeks). Tolerance was good and adverse effects, including alopecia, grade 1 epithelitis, and grade 2 leukopenia-anemia, were few. Sorafenib treatment was not authorized as a compassionate use by the patient's health insurance company (Fig. 2, right).

Six months after treatment, he was evaluated as partial response, but the reduction in the size of the tumor mass was insufficient to allow a complete surgical resection. He was included in a clinical trial with lenvatinib. Despite stabilization of tumor, the patient died three months later due to community-acquired pneumonia without leukopenia.

Patient 3. A 37-year-old male presented in the ER because of dysphonia, dyspnea and stridor due to a left laterocervical mass that had grown rapidly in 3 months. He was diagnosed with PTC with unresectable infiltration of the thyroideal cartilage, close to organs such as the trachea, esophagus, and skin (T4aN1M0). A PET-CT scan revealed a mass in the left thyroid lobe with intense uptake (SUV 6.9). A cervical MRI confirmed a bulky left cervical mass with tracheal compression and invasion of the surrounding skin (Fig. 3, left). External radiotherapy (64-Gy) and weekly doxorubicin $\left(20 \mathrm{mg} / \mathrm{m}^{2} \mathrm{IV}\right.$ for 8 weeks) with sorafenib (400 mg every $12 \mathrm{~h}$ p.o.) were administered concomitantly.

After 13 days receiving sorafenib $800 \mathrm{mg} /$ day, the patient presented adverse effects with grade 2 erythema and facial edema requiring dose adjustments (first $600 \mathrm{mg} / \mathrm{day}$ for 2 weeks and subsequently to $400 \mathrm{mg} / \mathrm{day}$ ), and subsequently grade 3 dermal toxicity and mucositis/folliculitis and grade 3 headache.

After 8 weeks of doxorubicin and 25 weeks of sorafenib, response was noted, with reduction in tumor size, decrease in thyroglobulin (TG) $177 \mathrm{ng} / \mathrm{ml}$ (basal serum level 1,090 ng/ml), and radiological partial response (Fig. 3,right). Adverse treatment effects were grade 3 lymphopenia, grade 2 mucositis/folliculitis and grade 3 headache.

Total thyroidectomy and cervical lymphadenectomy were subsequently performed. The pathological report revealed a PTC, $65 \mathrm{~mm}$ in size, macrofollicular, with fibroadipose invasion of extrathyroidal tissue, no vascular-neural invasion, and surgical margins of $1 \mathrm{~mm}$. Lymphadenectomy was performed showing 2 positive from 7 resected lymph nodes in area $\mathrm{V}$ and 1 positive from 5 resected lymph nodes in area VI.

Nine months after the operation, the patient remained asymptomatic. His voice was restored and he was pending radioiodine administration.

Patient 4. A 62-year-old woman with a clinical history of hypertension and multinodular goiter 3 years previously. In the last 2 months, the goiter had increased and left cervical lymphadenopathy appeared. She developed progressive dyspnea and hemoptysis. Cervicothoracic CT identified thyroid neoplasia with tracheal infiltration of up to $50 \%$ of the lumen in the proximal third (Fig. 4, left) and presence of lung metastases.

FNA reported FTC, and cervicotomy was performed showing large tumor extension from the cricoid cartilage to the sternal notch and infiltration of the tracheal rings, so the tumor was considered unresectable. Tracheal infiltration was confirmed by bronchoscopy, requiring tracheal prosthesis that 
Table I. Main toxicities and adverse events by grade (G) in each patient.

Toxicities/adverse

events

Case 1 Case 2 Case 3 Case 4 Case 5

$\begin{array}{lll}\text { Anemia } & \text { G2 } & \text { G2 } \\ \text { Leukopenia } & & \text { G2 }\end{array}$

Lymphopenia

Cutaneous-mucous

$\begin{array}{lll}\text { Mucositis } & \text { G2 } & \text { G2-3 } \\ \text { Folliculitis } & & \text { G2-3 }\end{array}$

Esophagitis

G2

Alopecia

G1

G1

Epitheliopathy

Skin Rash

Metabolic

Palmoplantar

erythrodysesthesia

Kidney Infection

Hypocalcemia

Asthenia

G1

G1

Neurologic

Migraine

Odynophagia

Gastrointestinal

Vomits

G1

Dysphonia

\section{Hematological}

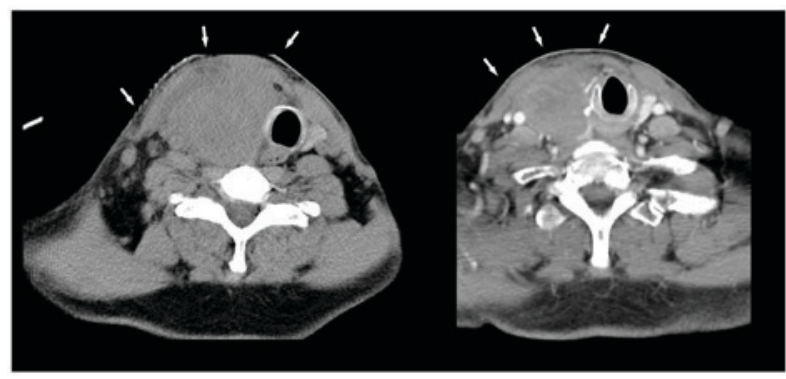

Figure 2. Computed tomography scan of Patient 2. Left panel: Showing a 60x94x68 mm right cervical mass (arrows). Right panel: Same patient after 50 Gy external radiotherapy concomitantly with doxorubicin $\left(20 \mathrm{mg} / \mathrm{m}^{2}\right.$ per week for 6 weeks).

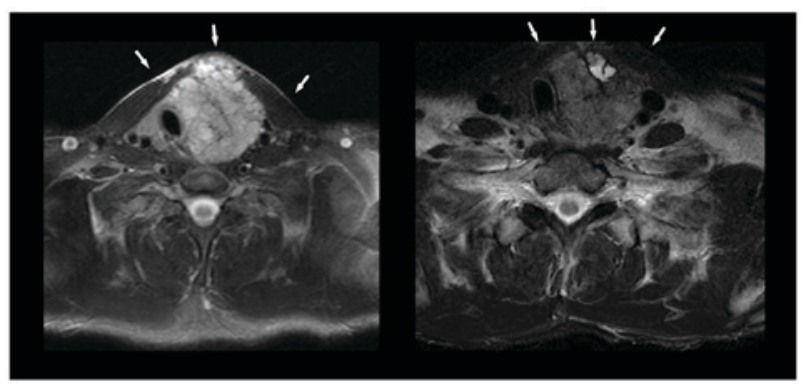

Figure 3. Cervical magnetic resonance imaging of Patient 3. Left panel: Showing an intense uptake (SUV 6.9) mass in the left thyroid lobe (arrows). Right panel: After 8 weeks with doxorubicin and 25 weeks with sorafenib and 64 Gy radiotherapy, a new MRI showed a partial response.

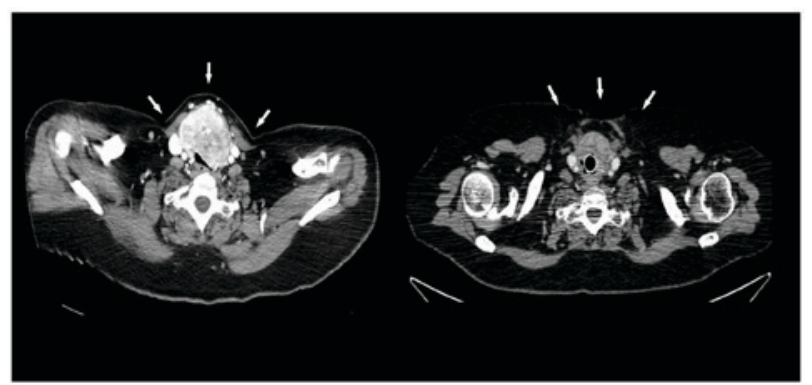

Figure 4. Positron emission tomography/computed tomography scan of Patient 4. Left panel: Showing a left cervical mass showing large tumor extension from the cricoid cartilage to the sternal notch and infiltration of tracheal rings (arrows). Right panel: Same patient after sorafenib $400 \mathrm{mg} / 12 \mathrm{~h}$ and radiotherapy TD $50 \mathrm{~Gy}$. A partial response of the left cervical mass was observed.

Figure 1. Positron emission tomography/computed tomography scan of Patient 1. Left panel: Showing a bulky left cervical mass compressing the trachea (arrows). Right panel: Same patient after treatment with 70 Gy palliative external radiotherapy, doxorubicin $\left(20 \mathrm{mg} / \mathrm{m}^{2}\right.$ per week) for 4 weeks, and sorafenib $(400 \mathrm{mg} / 12 \mathrm{~h})$ showing a partial response of the left cervical mass (arrows).

was placed and relocated after rejection; open biopsy of the lesion confirmed FTC.

Sorafenib $400 \mathrm{mg} / 12 \mathrm{~h}$ p.o. and radiotherapy TD $50 \mathrm{~Gy}$ were administered, showing evidence of clinical and serological response. TG levels were $230 \mathrm{ng} / \mathrm{ml}$ (baseline, $2,153 \mathrm{ng} / \mathrm{ml}$ ). A repeat CT showed partial response (Fig. 4, right). Treatment toxicity consisted of grade 1 hand-foot syndrome, hoarseness, asthenia, and nausea/vomiting and grade 2 sore throat. A significant partial response ( $>50 \%$ of primary tumor volume) was obtained after sorafenib plus radiotherapy, and this effect was maintained with sorafenib at standard doses. While being evaluated for salvage surgery, the patient presented a tumor infection in the context of significant tumor necrosis, producing uncontrolled bleeding and finally causing death.

Patient 5. A 61-year-old male, former smoker with history of acute glomerulonephritis $\operatorname{Ig} \mathrm{A}$ was seen in the outpatient facility due to asthenia, anorexia and weight loss, and diarrhea. Cervical ultrasound identified left thyroid nodule and cervical nodes. A biopsy of the cervical mass was performed and medullary thyroid carcinoma was diagnosed. 


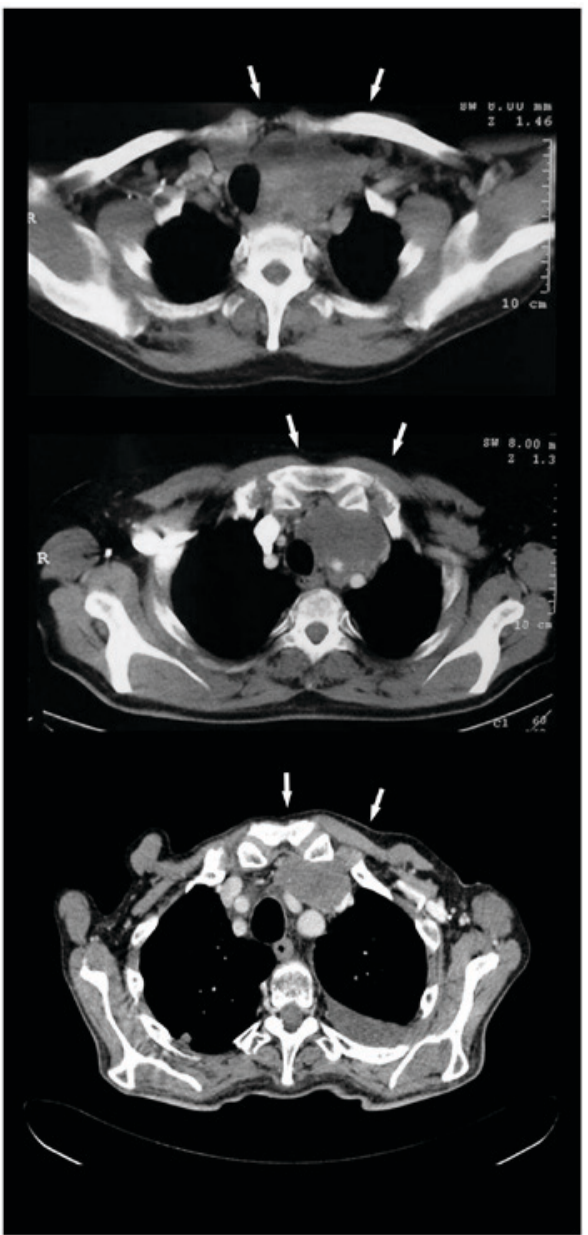

Figure 5. Cervicothoracic computed tomography of Patient 5. Upper panel: Scan showing mediastinal mass $(9 \times 7 \times 8 \mathrm{~cm}$; arrows) compressing and displacing the thyroid gland, trachea, esophagus, brachiocephalic vein, internal jugular vein, associated with enlarged mediastinal lymph nodes. Central panel: After external radiotherapy $(64.8 \mathrm{~Gy})$ and oral sorafenib $400 \mathrm{mg} /$ day, a $16 \%$ reduction in cervical mass was observed. Bottom panel CT scan 4 years and 3 months after treatment showing a partial response of the mediastinal tumor mass.

Cervicothoracic CT scan showed a mediastinal mass (9x7x8 cm) compressing and displacing the thyroid gland, trachea, esophagus, brachiocephalic vein, internal jugular vein, associated with enlarged mediastinal lymph nodes (Fig. 5, top); calcitonin was 20,000 IU and CEA $123 \mathrm{ng} / \mathrm{ml}$. Lanreotide every 28 days was initiated.

After progression 5 months later, the patient started sorafenib (400 mg/12 h) on a compassionate use basis. Cervicothoracic CT a month later described a slight increase in thyroid mass to $9 \times 9 \times 7.5 \mathrm{~cm}$ and new mediastinal lymph nodes. Serum calcitonin level was 48,793 IU.

At that time, external RT, 64.8 Gy TD was administered concomitantly with oral sorafenib (compassionate use) $400 \mathrm{mg}$ /day p.o. Patient presented serological response with calcitonin 12,806 IU and CEA $107 \mathrm{ng} / \mathrm{ml}$; reassessment with CT showed a $16 \%$ reduction in the size of the primary and mediastinal lymph nodes (Fig. 5, middle). Toxicity consisted of grade 1 palmoplantar erythrodysesthesia and grade 1 asthenia.

Sorafenib at $400 \mathrm{mg} / 12 \mathrm{~h}$ p.o. continued for 8 more months. At that time, the patient showed serological partial response and 30-40\% reduction of tumor mass according to mediastinal nodal response on CT (Fig. 5, bottom). This response was maintained for 4 years and 3 months when serological progression, calcitonin 24,000 IU and CEA $83 \mathrm{ng} / \mathrm{ml}$ were observed. At that time, he began vandetanib $300 \mathrm{mg} /$ day p.o. After a 3-month period with stable liver disease, adenopathic, pericardial, pleural, pulmonary and liver progression was observed causing death a few weeks later due to tumor progression.

\section{Discussion}

This is the first short series of 5 consecutive patients to appear on the tolerance, toxicity and therapeutic activity of the association of EBRT with systemic therapy with doxorubicin or sorafenib in locally advanced thyroid cancer.

The first impression of these clinical results is that the treatments can be combined with no excessive increase in toxicity and that the antitumor activity has been acceptably good. According to the ATA, EBRT in patients with DTC is indicated in cases of bulky unresectable disease (both primary and local recurrence) and in other situations, such as high risk of local recurrence, residual disease post-thyroidectomy, tumor without $\mathrm{I}^{-131}$ uptake, postoperative serum TG levels $\geq 1 \mathrm{ng} / \mathrm{ml}$, and in select patients $>45$ years old with high likelihood of microscopic residual disease (2).

Patients with dyspnea and stridor usually present a serious clinical challenge and they have short life expectancy of a few weeks. In this situation, EBRT is sometimes the only therapeutic option (4-6). However to date, the use of EBRT in DTC has not been supported by randomized studies, probably since recruitment is limited because of the low incidence of this entity and the good response to $\mathrm{I}^{-131}$ in most cases (3-5). In the past, the only palliative systemic drug of choice for treatment of DCT was doxorubicin (5). Nevertheless, it is not currently recommended in the clinical guidelines for use in monotherapy because of its low efficacy and high toxicity (14). Given the evidence on the concomitant use of radiation therapy with doxorubicin in other bulky tumors (such as anaplastic thyroid carcinoma) (15), we decided to administer the same treatment regimen with EBRT and weekly doxorubicin at metronomic doses in patients with DTC and bulky disease. Treatment was sometimes administered in conjunction with sorafenib, and the double drug therapy combined with EBRT was not well tolerated by the patient (Table I, Patient 3 ).

In general, tolerance was acceptable, with few side effects and a low grade of hematological toxicity, occurring mainly when doxorubicin was associated with radiotherapy. Other authors also suggest that the combination of sorafenib should not increase hematological toxicity, and the other side effects described in our patients were mainly cutaneous (16). Therapeutic efficacy seems to be acceptable because symptomatic control of dyspnea and stridor was achieved in all 5 patients. Furthermore, salvage surgery was performed in one patient, resulting in local control of the disease. In patient 1 , the switch to lenvatinib after disease progression at 6 months of follow up may have contributed to his long survival.

These results suggest that the combination of EBRT with sorafenib has good efficacy with an acceptable toxicity profile. It is important to take into account the high percentage of locoregional complications in patients with locally advanced thyroid 
cancer when radical combined therapy is applied, as rapid tumor necrosis can cause fistulae or serious local problems.

These clinical results are limited by the small sample size of our series. From a mechanistic point of view, several studies support the notion that the anti-angiogenic effect of sorafenib might enhance the antitumor activity of radiation therapy through angiogenesis inhibition, endothelial cell radiosensitivity, tumor cell apoptosis, or a decrease in the number of hypoxic cells (improved oxygenation) $(17,18)$. Nevertheless, these same mechanisms may be responsible for increased radiation toxicity, mainly causing mucositis and skin rash (18).

More information must be generated with retrospective or prospective studies to confirm the good tolerance and antitumor activity of the combination of external radiotherapy with new anti-target agents such as sorafenib and doxorubicin in DTC.

\section{Acknowledgements}

Not applicable.

\section{Funding}

No funding was received.

\section{Availability of data and materials}

The datasets used and/or analyzed during the current study are available from the corresponding author on reasonable request.

\section{Authors' contributions}

JJG and JC collaborated in the conception and design of the present study. JJG, JC and FR collected and assembled the data. JJG, KSCM and KH collected and assembled the data. JJG and JC were involved in data analysis and interpretation. All authors contributed to writing the manuscript and approved the final version.

\section{Ethics approval and consent to participate}

Approval was obtained from the Clinical Research Ethics Committee at Hospital Clínic of Barcelona's (fclinic@clinic.ub.es) Reg. HCB/2018/00067.

\section{Patient consent for publication}

According Spanish law, informed written consent was obtained from patients still alive for publication of the data in the present study.

\section{Competing interests}

The authors declare that they have no competing interests.

\section{References}

1. Howlader N, Noone AM, Krapcho M, Miller D, Bishop K Altekruse SF, Kosary CL, Yu M, Ruhl J, Tatalovich Z, et al (eds): SEER Cancer Statistics Review, 1975-2013. National Cancer Institute, Bethesda, MD. https://seer.cancer.gov/csr/1975_2013/. Accessed September 12, 2016.
2. Haugen BR, Alexander EK, Bible KC, Doherty GM, Mandel SJ, Nikiforov YE, Pacini F, Randolph GW, Sawka AM, Schlumberger M, et al: 2015 American Thyroid Association management guidelines for adult patients with thyroid nodules and differentiated thyroid cancer: The American Thyroid Association guidelines task force on thyroid nodules and differentiated thyroid cancer. Thyroid 26: 1-133, 2016.

3. Schwartz DL, Lobo MJ, Ang KK, Morrison WH, Rosenthal DI, Ahamad A, Evans DB, Clayman G, Sherman SI and Garden AS: Postoperative external beam radiotherapy for differentiated thyroid cancer: Outcomes and morbidity with conformal treatment. Int J Radiat Oncol Biol Phys 74: 1083-1091, 2009.

4. Terezakis SA, Lee KS, Ghossein RA, Rivera M, Tuttle RM, Wolden SL, Zelefsky MJ, Wong RJ, Patel SG, Pfister DG, et al: Role of external beam radiotherapy in patients with advanced or recurrent nonanaplastic thyroid cancer: Memorial Sloan-kettering cancer center experience. Int J Radiat Oncol Biol Phys 73: 795-801, 2009.

5. Gottlieb JA and Hill CS Jr: Chemotherapy of thyroid cancer with adriamycin: Experience with 30 patients. N Engl J Med 290: 193-197, 1974.

6. Kim JH and Leeper RD: Combination adriamycin and radiation therapy for locally advanced carcinoma of the thyroid gland. Int J Radiat Oncol Biol Phys 9: 565-567, 1983.

7. Kim JH and Leeper RD: Treatment of locally advanced thyroid carcinoma with combination doxorubicin and radiation therapy. Cancer 60: 2372-2375, 1987.

8. Rosenthal CJ and Rotman M: Pilot study of interaction of radiation therapy with doxorubicin by continuous infusion. $\mathrm{NCI}$ Monogr: 285-290, 1988.

9. Welsh JW, Komaki R, Amini A, Munsell MF, Unger W, Allen PK, Chang JY, Wefel JS, McGovern SL, Garland LL, et al: Phase II trial of erlotinib plus concurrent whole-brain radiation therapy for patients with brain metastases from non-small-cell lung cancer. J Clin Oncol 31: 895-902, 2013.

10. Sano D, Matsumoto F, Valdecanas DR, Zhao M, Molkentine DP, Takahashi Y, Hanna EY, Papadimitrakopoulou V, Heymach J, Milas L and Myers JN: Vandetanib restores head and neck squamous cell carcinoma cells' sensitivity to cisplatin and radiation in vivo and in vitro. Clin Cancer Res 17: 1815-1827, 2011.

11. Brose MS, Nutting CM, Jarzab B, Elisei R, Siena S, Bastholt L, de la Fouchardiere C, Pacini F, Paschke R, Shong YK, et al: Sorafenib in radioactive iodine-refractory, locally advanced or metastatic differentiated thyroid cancer: A randomised, double-blind, phase 3 trial. Lancet 384: 319-328, 2014.

12. Capdevila J, Iglesias L, Halperin I, Segura A, Martínez-Trufero J, Vaz MÁ, Corral J, Obiols G, Grande E, Grau JJ and Tabernero J: Sorafenib in metastatic thyroid cancer. Endocr Relat Cancer 19: 209-216, 2012

13. Eisenhauer EA, Therasse P, Bogaerts J, Schwartz LH, Sargent D, Ford R, Dancey J, Arbuck S, Gwyther S, Mooney M, et al: New response evaluation criteria in solid tumours: Revised RECIST guideline (version 1.1) Eur J Cancer 45: 228-247, 2009.

14. Pacini F, Castagna MG, Brilli L and Pentheroudakis G; ESMO Guidelines Working Group: Thyroid cancer: ESMO clinical practice guidelines for diagnosis, treatment and follow-up. Ann Oncol 23 (Suppl 7): vii110-vii119, 2012.

15. Sherman EJ, Lim SH, Ho AL, Ghossein RA, Fury MG, Shaha AR, Rivera M, Lin O, Wolden S, Lee NY and Pfister DG: Concurrent doxorubicin and radiotherapy for anaplastic thyroid cancer: A critical re-evaluation including uniform pathologic review. Radiother Oncol 101: 425-430, 2011.

16. Brierley J and Sherman E: The role of external beam radiation and targeted therapy in thyroid cancer. Semin Radiat Oncol 22: 254-262, 2012.

17. Lee CG, Heijn M, de Tomasso E, Griffon-Etienne G, Ancukiewicz M, Koike C, Park KR, Ferrara N, Jain RK, Suit HD and Boucher Y: Anti-Vascular endothelial growth factor treatment augments tumor radiation response under normoxic or hypoxic conditions. Cancer Res 60: 5565-5570, 2000.

18. Murray L, Longo J, Wan J, Chung C, Wang L, Dawson L, Milosevic M, Oza A and Brade A: Phase I dose escalation study of concurrent palliative radiation therapy with sorafenib in three anatomical cohorts (Thorax, Abdomen, Pelvis): The TAP study. Radiather Oncol 124: 74-79, 2017. 